\title{
Experiencias de voluntariado en la CAPV
}

\section{Dirección de Política Familiar y Comunitaria, Dpto. de Empleo y Asuntos Sociales, Eusko Jaurlaritza-Gobierno Vasco}

<familia@ej-gv.es>

Gertu dago Boluntariotzaren Europako Urtearen bukaera, eta testu honek Euskal Autonomia Erkidegoan esparru ezberdinetan garatzen diren laguntzarako ekintzen zenbait adibide erakutsi nahi ditu: DYAren errepideko laguntza, Gurutze Gorriaren akonpainamendu zerbitzua eta Euskadiko laguntzarako txakurren taldea. Gaur egungo testuinguru zailean, boluntariotzaren laguntza handia izan daiteke biztanleriaren bizi-kalitatea hobetzeko, eta boluntarioek egiten duten lanak esker ona merezi du hartan jartzen duten konpromiso eta ardurarengatik.

\section{HITZ-GAKOAK:}

boluntariotza, motibazioa, irabazi asmorik gabeko elkarteak, esperientziak, laguntza.
A punto de que termine la celebración del Año Europeo del Voluntariado, este texto muestra algunos ejemplos de actividades de ayuda que se desarrollan en distintos ámbitos en la CAPV y que cuentan principalmente con personal no remunerado: la Asociación de Ayuda en Carretera DYA, el Programa de Acompañamiento de la Cruz Roja y el Grupo del Perro de Salvamento de Euskadi. En un contexto social difícil, el voluntariado constituye una importante fuente de ayuda para mejorar la calidad de vida de la población, y la labor que realizan las personas voluntarias merece un reconocimiento, por su dedicación y compromiso.

\section{PALABRAS ClaVe:}

voluntariado, motivación, entidades sin ánimo de lucro, experiencias, apoyo.

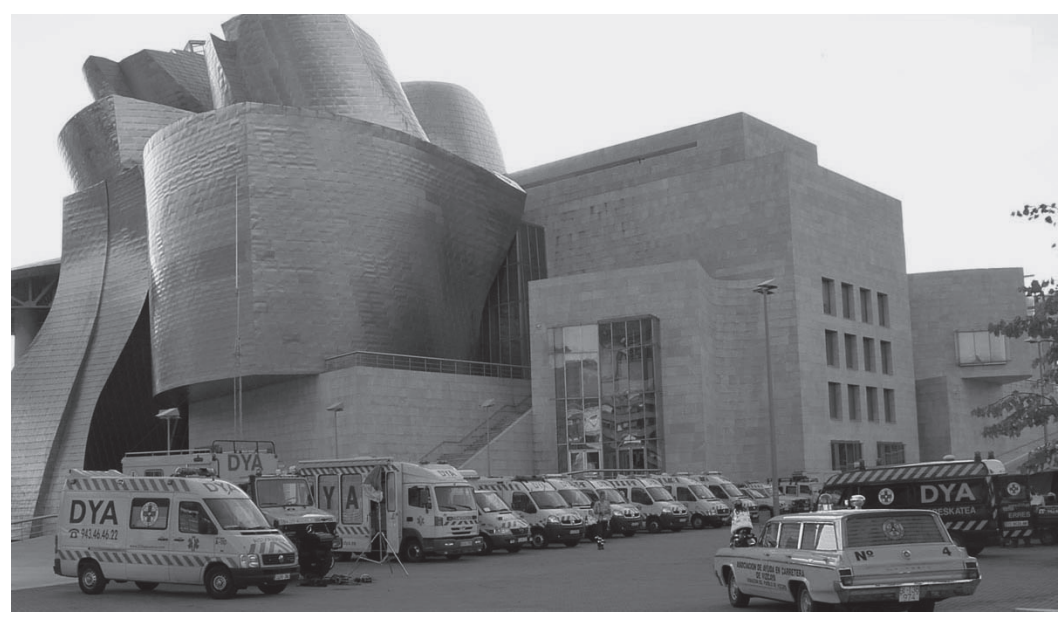

Ambulancias de la DYA en el exterior del Museo Guggenheim.

Fuente: Asociación de Ayuda en Carretera DYA. 


\section{Introducción}

La Ley 17/1998 del Voluntariado de la CAPV define el voluntariado como el conjunto de actividades de interés general desarrolladas por personas físicas, siempre que se realicen en las siguientes condiciones: de manera desinteresada y con carácter solidario; voluntaria y libremente, sin traer causa de una relación laboral, funcionarial o mercantil, o de una obligación personal o deber jurídico; a través de organizaciones sin ánimo de lucro, y con arreglo a programas o proyectos concretos; sin retribución económica y sin sustituir, en ningún caso, servicios profesionales remunerados. Con respecto a las actividades de interés general, son aquellas que comportan un compromiso en favor de la sociedad o de la persona y que se desenvuelven tanto en el ámbito social, comunitario, cívico, cultural, de cooperación al desarrollo, de protección al medio ambiente o cualquier otro de naturaleza análoga. Dentro del voluntariado, por lo tanto, puede hablarse de una gran diversidad de actividades y de ámbitos de actuación.

Este 2011 ha sido el año Europeo del Voluntariado, en el que han participado todos los Estados miembros, y que ha convocado en toda Europa multitud de exposiciones, programas y actividades de todo tipo. Para estos doce meses, el Parlamento Europeo se marcó, como objetivos principales (Comisión Europea, 20102011), trabajar para lograr un entorno que favorezca el voluntariado, ofrecer medios de actuación a las organizaciones de voluntarios con el fin de ayudarles a poner en marcha nuevos tipos de acciones voluntarias, y propiciar el establecimiento de redes y cooperación con la sociedad civil.

El voluntariado es una actividad fundamental en todas las entidades sin ánimo de lucro, y una expresión de participación social que las enriquece y les ofrece un valor añadido. Sin embargo, algunas organizaciones han visto reducirse el número de personas voluntarias en los últimos años. Parece que, entre los principales obstáculos para atraer a nuevas personas voluntarias, están la coyuntura de crisis económica, las crecientes dificultades para conciliar la vida laboral, familiar y personal, y también los cambios que se están produciendo en el sistema de valores. En cuanto a las dificultades para mantener al voluntariado en las organizaciones, hay que destacar algunos factores relacionados con el desarrollo organizativo (crecimiento desordenado o rápido, procesos de profesionalización), igual que la forma de gestionar el voluntariado en las organizaciones (carencias en el seguimiento y el apoyo al personal voluntario, escasa formación y falta de programas de captación, entre otras).

El último informe anual del Gobierno Vasco sobre la situación del voluntariado en la CAPV (2009) afirma que las organizaciones de voluntariado en el territorio tienen, en su gran mayoría, más de cinco años de antigüedad $(93,2 \%)$, lo que indica que se trata de organizaciones con experiencia. La mayoría $(81,8 \%)$ son asociaciones, el $58 \%$ de ellas son organizacio- nes especializadas y un $71 \%$ desarrollan su trabajo voluntario a favor de otras personas. Con respecto al voluntariado que participa en estas organizaciones, según el informe, la mayoría de éstas cuentan con un voluntariado sin límite de edad (77,6\%). En el 89,4\% de las entidades el voluntariado representa más del $50 \%$ del total de personas que intervienen en ellas, y en la mayoría de casos el personal voluntario supera al remunerado. La mayor parte de las organizaciones tienen entre 10 y 50 personas voluntarias, con una presencia algo superior del voluntariado femenino con respecto al masculino. El grupo de edad que predomina entre el voluntariado va de los 18 a los 45 años, y su labor tiene, en general, carácter estable (una media de más de 6 horas semanales). Sólo el $6,8 \%$ realiza tareas directivas en la organización.

Como ya hemos señalado, los tipos de voluntariado que pueden realizarse son de muy diversa índole. En la CAPV, en concreto, y según el informe antes mencionado, la actividad del personal voluntario se centra preferentemente en la atención a la población en general; a niños/as, adolescentes y jóvenes; y a las mujeres, en este orden. Este artículo es el primero de una serie que se encargará de recoger las diferentes experiencias de voluntariado que se dan en la comunidad autónoma de Euskadi, tanto dentro como fuera de los servicios sociales, con la intención de mostrar el valor del trabajo que realizan estas personas para el bienestar de la sociedad, y la heterogeneidad de los servicios a los que dedican su tiempo y su esfuerzo.

\section{Asociación de Ayuda en Carretera (DYA)}

La Asociación de Ayuda en Carretera (DYA) nació en 1966, con el objetivo de tratar de forma humana a las víctimas de accidentes de tráfico, dado que en ese momento no había ambulancias para atender a las personas accidentadas. Todos los voluntarios que participan en ella tienen el título de socorrista, y quienes no lo tienen van con personas experimentadas mientras se preparan para obtenerlo. Para conducir ambulancias, es preciso tener al menos de 21 años y adquirir una formación previa, además de otros requisitos.

La asistencia que proporciona la asociación consiste en la ayuda de primeros auxilios: explorar, estabilizar, frenar hemorragias o entablillar, entre otras tareas. Si la urgencia lo requiere, se realiza el soporte vital básico con maniobras de reanimación, siempre que la víctima esté inconsciente, tenga comprometida la respiración y la circulación cardiovascular, y no sea posible esperar a que llegue otra ambulancia al lugar de los hechos. Aun así, en la asociación tienen claro que una persona voluntaria no puede hacer más de lo que le permiten sus conocimientos, así que cuando la atención requerida es más específica, se cuenta con ayuda suplementaria, como una ambulancia de soporte vital avanzada con un médico propio. Actualmente, además de la ayuda en carretera, la asociación también trabaja en la sensibilización y la 
educación vial, puesto que considera que la educación y la prevención son las principales herramientas para evitar los riesgos del tráfico sin recurrir a las sanciones.

\section{Programa de Acompañamiento de Cruz Roja}

La Cruz Roja española nació a mediados del siglo XIX con el fin de prestar auxilio a los heridos en conflictos armados. Desde entonces, se ha ido adaptando a las necesidades que se iban detectando en la sociedad, desde la atención a las madres de familias desfavorecidas en la década de 1920 , a reunir a familias durante la Guerra Civil, o a promover la prevención de la diabetes, hasta llegar a la década de los noventa, cuando se produjo una gran modernización de la entidad, que se adaptó a nuevos retos: consolidar la intervención social con los colectivos vulnerables (personas mayores, inmigrantes y refugiados, personas afectadas por VIH, drogodependientes, infancia y juventud, discapacidad, mujeres o población reclusa), aumentar los programas internacionales, incorporar el medio ambiente como un ámbito más de actuación y utilizar las nuevas vías de colaboración surgidas gracias a las nuevas tecnologías. El objetivo del programa de acompañamiento, que se puso en marcha en 1996, es paliar la soledad de personas mayores que viven en residencias o en sus domicilios particulares. La labor del personal voluntario no consiste en cubrir necesidades básicas, como la higiene personal o la del entorno de la persona mayor, sino su falta de compañía. El voluntariado dedica entre una hora y media y dos horas semanales a realizar con ellos sus actividades preferidas (pasear, charlar, leer el periódico, hacer gestiones o jugar a cartas). Además, merece la pena notar que el programa se interrelaciona con otros proyectos, como el de telealarma, ya que las visitas a las personas mayores en sus domicilios sirven para valorar los casos que son susceptibles de necesitar el servicio.

Los voluntarios reciben formación específica por parte de cruz Roja para atender a las necesidades de las personas mayores de la manera más adecuada, pero la organización no les exige requisitos específicos para participar en el programa, siempre que tengan más de 18 años y disfruten estando con personas mayores. Cualquier persona puede aportar su experiencia, sus conocimientos y su tiempo. Además de un elevado número de proyectos, la organización también tiene la capacidad y la experiencia de desarrollar nuevos programas ideados por la propia institución, o que nacen de las ideas que aporta el personal voluntario.

El voluntariado de acompañamiento puede vivir situaciones muy diversas, asistiendo a personas que no pueden hablar debido a que tienen parálisis cerebral, a personas que necesitan ayuda para poder moverse 0 a otras que no tienen a nadie con quien compartir una conversación. Esas dos horas que pasan con el voluntariado son muy valiosas para las

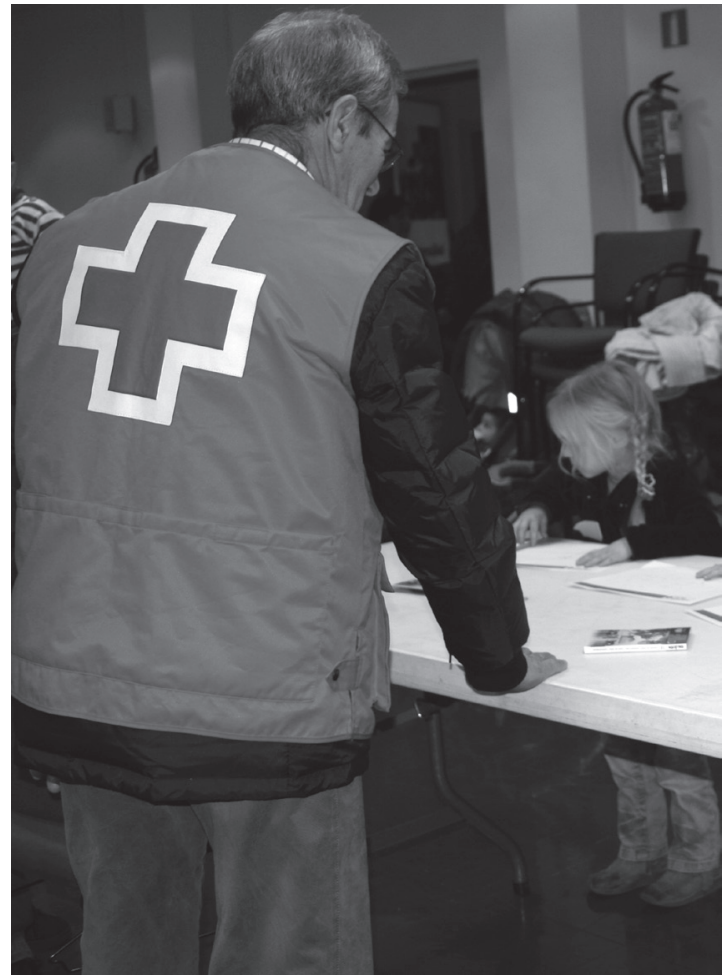

Voluntario de la Cruz Roja en una actividad con niños y niñas. Fuente: Cruz Roja.

personas acompañadas. Porque, aunque es evidente que la sociedad ha mejorado en lo que respecta a la preocupación por la salud y las comodidades de las personas mayores, la soledad sigue estando muy presente en el colectivo atendido.

\section{Grupo del Perro de Salvamento de Euskadi}

El Grupo del Perro de Salvamento de Euskadi se creó en 1983 , con el objetivo principal de preparar equipos formados por un guía y un perro, destinados a localizar personas perdidas en el monte, sepultadas entre escombros o enterradas bajo la nieve. Con sede en Elorrio (Bizkaia), la asociación está formada por personas voluntarias, que prestan un servicio de 24 horas al día los 365 días del año. Esta disponibilidad se consigue gracias a que el voluntariado negocia con las empresas la posibilidad de ausentarse del puesto de trabajo en caso de activación para una búsqueda, y recuperar esas horas más delante, pero sobre todo gracias al apoyo de sus familiares y amistades, que son quienes más sufren las ausencias de las personas voluntarias, debidas a los entrenamientos, las intervenciones y demás aspectos relacionados con la actividad del grupo.

Empiezan a trabajar con los perros a los tres meses de su nacimiento, en el momento del destete. El entrenamiento responde a diversas pautas y métodos fijados dentro del grupo, para que, hacia los dos años, el perro esté equilibrado y preparado para 
empezar a trabajar en rescates. Aun así, el trabajo de los equipos no termina nunca. Se realizan tres entrenamientos en grupo a la semana, que suponen unas diez horas, además del entrenamiento personal de cada equipo (persona y perro) y la actividad cotidiana con el animal, que incluye los paseos o la práctica de la obediencia. Además del tiempo invertido en el entrenamiento, la asociación tiene un compromiso firmado con el Gobierno Vasco para homologar a todos los equipos por lo menos una vez al año.

Con respecto a los rescates, la mayor parte de las movilizaciones se realizan a través de SOS Deiak, que informa al responsable de intervención del grupo sobre la situación en la que deben intervenir. $\mathrm{El}$ grupo valora las circunstancias, y activa a los equipos y personas de apoyo que considera necesarios. Siempre hay una persona de SOS Deiak como responsable de la búsqueda, que es la encargada de adjudicar la zona de trabajo a los equipos. La labor de los voluntarios es batir con los perros la zona que les han indicado y descartarla si la persona desaparecida no está en ella.

Este tipo de voluntariado requiere mucho esfuerzo, puesto que desde que se empieza a trabajar con un perro hasta que llega a ser operativo en rescate pasan dos años, como mínimo, e incluso puede no llegar a estarlo nunca. Por eso, llega mucha gente nueva al grupo, pero son pocos los que se quedan. Quienes lo hacen afirman que su principal motivación es ser amantes de los perros, pero también valoran los profundos lazos de amistad que se crean entre personas que comparten infinidad de momentos, tanto buenos como malos, y la sensación de enriquecimiento personal que proporciona un servicio a la sociedad.

\section{A modo de cierre}

Éstos son sólo tres ejemplos de las numerosas acciones de voluntariado desarrolladas en la CAPV, que muestran que la actividad voluntaria ofrece enormes satisfacciones, pero también requiere esfuerzo y compromiso. A fin de no perder voluntariado y conseguir a más personas que quieran colaborar con ellas, las organizaciones deben identificar nuevas fórmulas de captación y seguimiento, que estén bien organizadas y sigan un proceso estructurado. En lo referente a la sensibilización, es importante que las entidades cuenten con una persona encargada de comunicación, que planifique adecuadamente la estrategia, para poder llegar a la sociedad y al voluntariado potencial.

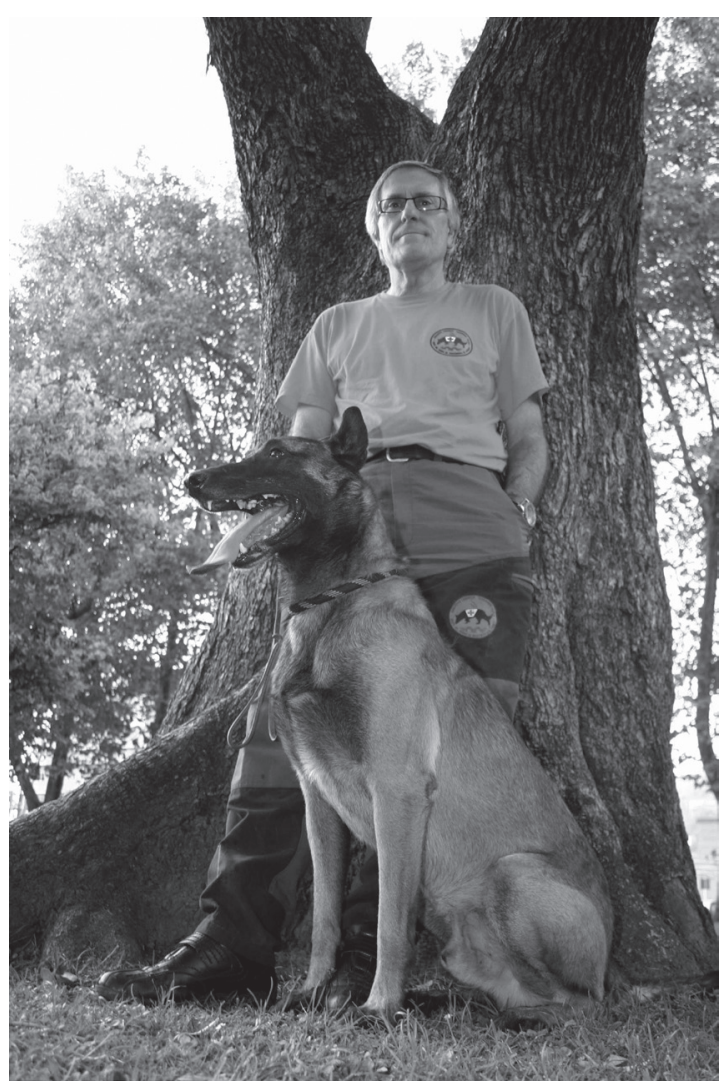

Un equipo del Grupo del Perro de Salvamento de Euskadi, formado por un voluntario y su perro.

Fuente: Grupo del Perro de Salvamento de Euskadi.

\section{Referencias bibliográficas}

COMISIÓN EUROPEA (2010-2011): Año Europeo del Voluntariado 2011 [rhttp://europa.eu/ volunteering/es/ $/$ ].

GOBIERNO VASCO (2010): Informe anual de la situación de las organizaciones de voluntariado en la CAPV 2009, Vitoria-Gasteiz, Eusko JaurlaritzaGobierno Vasco ['http://goo.gl/k8FtO〉]. 